\title{
"Make the Implicit Explicit": Measuring Perceptions of Gender Bias and Creating a Gender Bias Curriculum for Internal Medicine Residents
}

This article was published in the following Dove Press journal:

Advances in Medical Education and Practice

\author{
Paula Chatterjee (D) \\ Lindsay N Warner ${ }^{2}$ \\ Maria C Basil \\ Michelle Christopher ${ }^{3}$ \\ Katharine Manning ${ }^{4}$ \\ Herrick N Fisher ${ }^{4}$ \\ Kathryn M Rexrode ${ }^{4}$ \\ Sonja R Solomon ${ }^{4}$ \\ Rose M Kakoza ${ }^{5}$ \\ Maria A Yialamas ${ }^{4}$ \\ 'Department of Medicine, Perelman \\ School of Medicine at the University of \\ Pennsylvania, Philadelphia, PA, USA; \\ ${ }^{2}$ Department of Medicine, Virginia Mason \\ Medical Center, Seattle, WA, USA; \\ ${ }^{3}$ Department of Medicine, Tulane \\ University School of Medicine, New \\ Orleans, LA, USA; ${ }^{4}$ Department of \\ Medicine, Brigham \& Women's Hospital, \\ Boston, MA, USA; ${ }^{5}$ Department of \\ Primary Care and Community Medicine, \\ Christiana Care, Wilmington, DE, USA
}

Background: Gender bias in clinical training has been well established; however, little is known about how perceptions differ between men and women. Furthermore, few curricular options have been developed to discuss gender bias.

Objective: To measure the prevalence of gender bias, examine qualitative differences between men and women, and create a gender bias curriculum for internal medicine residents.

Methods: We surveyed 114 residents (response rate of 53.5\%) to identify the prevalence and types of gender bias experienced in training. We compared estimates between genders and organized qualitative results into shared themes. We then developed a curriculum to promote and normalize discussions of gender bias.

Results: Among surveyed residents, $61 \%$ reported personal experiences of gender bias during training, with $98 \%$ of women and $19 \%$ of men reporting experiences when stratified by gender. We identified two domains in which gender bias manifested: role misidentification and a difficult working environment. Residents identified action items that led to the development of a gender bias curriculum. The curriculum includes didactic conferences and training sessions, a microaggression response toolkit, dinners for men and women residents, participation in a WhatsApp support group, and participation in academic projects related to gender bias in training.

Conclusion: We confirmed a wide prevalence of gender bias and developed a scalable curriculum for gender bias training. Future work should explore the long-term impacts of these interventions.

Keywords: graduate medical education, curriculum development, survey

\section{Plain Language Summary}

Understanding how medical trainees perceive gender bias in the workplace is important to their well-being and essential for addressing disparities in academic medicine. In this singlecenter survey, we found that gender bias is widely prevalent among trainees, and tends to be more prevalent among women than men trainees. Specifically, gender bias in residency training can manifest as role misidentification or a difficult working environment. We developed a scalable, multi-component curriculum that can be used to address instances of gender bias experienced in residency.

\section{Introduction}

Department of Medicine, Perelman School of Medicine at the University of Pennsylvania, 423 Guardian Drive, Room 1318, Philadelphia, PA 19|40, USA

$\mathrm{Tel}+$ I (2I5) 573-9468

Email pchat@pennmedicine.upenn.edu
The prevalence and impact of gender bias in clinical training ${ }^{1}$ and academic medicine ${ }^{2}$ have been well documented. ${ }^{3}$ Unconscious gender bias in the care of patients can adversely influence clinical reasoning, promote stereotyping, and 
support confirmation biases. ${ }^{4,5}$ Outside of clinical care and training, unconscious bias in academic medicine has been associated with the delayed promotion and lower salaries among women. ${ }^{6-8}$ Understanding how trainees perceive instances of gender bias has important implications for their well-being as well as potential downstream consequences for mitigating disparities in academic medicine.

In this study, we had two objectives: first, we performed a survey to understand the prevalence of gender bias as perceived by men and women internal medicine trainees, and examined qualitative differences in experiences between genders. Second, we developed a scalable, multimodal curriculum to introduce the topic of unconscious gender bias into graduate medical education.

\section{Methods}

We surveyed internal medicine residents at a large academic medical center in Boston, MA in February 2018. We collected anonymized demographic data on selfidentified gender identity, race, and ethnicity. We then asked whether residents had encountered personal experiences of gender bias during residency, and whether they had observed colleagues experiencing instances of gender bias. For those that had experienced gender bias, we offered an optional, anonymized free text response to describe specific circumstances. We also solicited residents for action items to better address gender bias among trainees. We stratified survey results by gender and compared them using Chi-Square tests with p-values significant at $<0.05$. On the basis of data collected from the survey, we developed a gender bias curriculum for internal medicine residents. The study was deemed exempt by the institutional review board (IRB) of Partners Healthcare, Boston, MA since the project was undertaken as a quality improvement project in the residency program. As such, formal supervision and informed consent as approved by the IRB were not required per their policies.

\section{Results}

We surveyed 213 internal medicine interns and residents of whom 114 responded to the survey $(53.5 \%$ response rate). Forty-six percent identified as men and 54\% as women. The majority of residents identified as White (75\%, Supplemental Table 1), and were of non-Hispanic, Latino, or Spanish origin (93\%).

Among surveyed residents, 61\% reported personal experiences of gender bias. Among female residents, $98 \%$ reported personal experiences of gender bias during residency, while 98\% reported observing resident colleagues experience gender bias (Figure 1). In contrast, 19\% of male residents reported personal experiences of gender bias, though 90\% reported observing resident colleagues experience gender bias. Comparisons between genders were statistically significant $(\mathrm{p}<0.001$ for both personal experiences and observations of resident colleagues experiencing gender bias).

We elicited two primary themes from the self-described instances of gender bias: role misidentification and a difficult working environment (Supplemental Table 2). Typical examples of role misidentification including women residents being referred to as nurses or other nonphysician allied health personnel. Examples of difficult working environment included women residents experiencing more frequent interruptions, and more questioning of clinical decision-making from team members and other hospital staff.

Residents expressed a desire to learn more about gender bias and identified multiple action items: making coresidents, faculty, nurses, and ancillary staff aware of gender bias through conferences or training; enhancing the environment to discuss gender bias without shaming individuals; teaching residents how to respond to experienced or witnessed biases; and recruiting more women faculty in teaching and leadership roles.

In response to feedback, we developed a genderfocused unconscious bias curriculum for residents with the following components: (1) a multidisciplinary didactic conference to review data on unconscious bias in health care; (2) a microaggression response toolkit to equip residents with skills to respond to moments of bias encountered on the wards; (3) voluntary biennial dinners for men and women residents to openly discuss issues of gender bias in training; (4) voluntary participation in a WhatsApp support group for residents who identify as women; and (5) voluntary participation in academic projects that measure gender bias within training which has resulted in scholarly publications under mentorship from residency program leaders. ${ }^{9,10}$

\section{Discussion}

Graduate medical education continues to grapple with multiple competing needs. Nonetheless, discussing unconscious bias, particularly as it pertains to gender, is increasingly important as professional disparities persist and grow in academic medicine. 


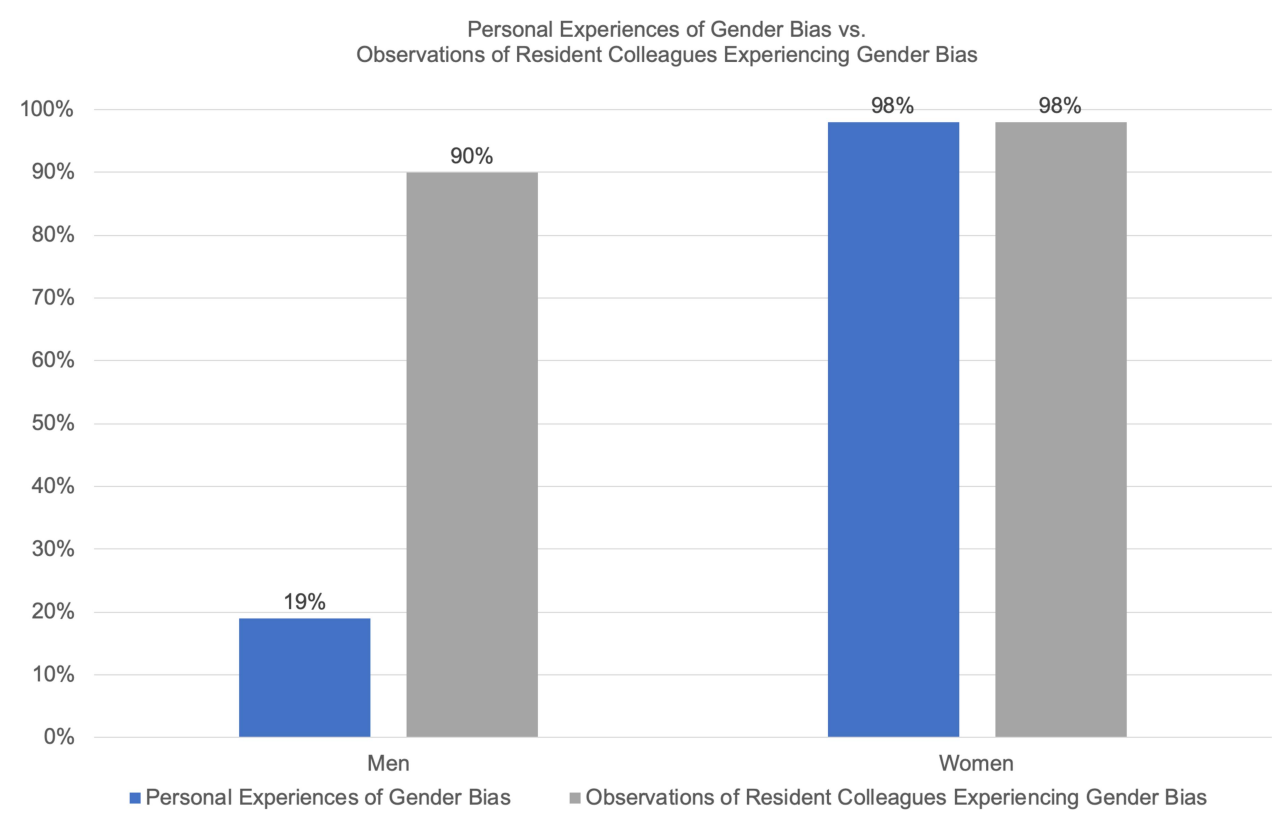

Figure I Prevalence of personal experiences of gender bias and observations of resident colleagues experiencing gender bias, stratified by gender.

Similar to prior work, we found a wider prevalence of perceived gender bias among women than men in our single-site survey but also found that men commonly observed their colleagues' experiences with such bias. These results suggest important differences in the perceived prevalence of gender bias between the level of the clinical trainee and that of faculty. Prior work has shown that approximately $70 \%$ of female junior faculty in academic medicine have perceived gender bias in the work environment, and a similar percentage has experienced it in their professional advancement; in contrast, little over $20 \%$ of male junior faculty perceived gender bias among their colleagues in the workplace. ${ }^{11}$ Our results confirm the wide prevalence of gender bias faced by trainees, ${ }^{1}$ but also suggest that male trainees more often detect such bias as compared to male faculty members. This may reflect growing efforts to incorporate unconscious bias education into medical school and residency. ${ }^{12}$ Few curricula have been developed to address unconscious bias in graduate medical education, though our work attempts to begin filling this gap. The gender bias curriculum described here remains in place for this residency program and has since been expanded to include an interactive workshop designed to improve residents' comfort with responding to microaggressions in the workplace. Current evaluations of these efforts are ongoing. ${ }^{13}$

Our study has limitations. We were limited to a single site for data collection, and we did not collect detailed information on the exact context of gender bias faced on the wards. Also, while we were focused on the role of gender bias, some of our qualitative results reflect the use of terminology consistent with sex instead of gender. Finally, given the recent implementation of the curriculum, we are unable to study how its introduction has changed the experiences of gender bias faced by residents during training.

\section{Conclusion}

Residency training is a formative time for a physician, and at this early stage of entering the profession, gender bias could have a large impact on career trajectories. Further research is needed to explore the settings in which trainees experience gender bias and how curricula can be designed to educate all those who take part in the clinical training process. In the future, potential interventions could be designed to reduce role misidentification and difficult work environments, as well as activate bystanders to mitigate adverse consequences.

\section{Author Contributions}

All authors made substantial contributions to conception and design, acquisition of data, or analysis and interpretation of data; took part in drafting the article or revising it critically for important intellectual content; agreed to submit to the current journal; gave final approval of the 
version to be published; and agree to be accountable for all aspects of the work.

\section{Disclosure}

The authors report no conflicts of interest in this work.

\section{References}

1. Fnais N, Soobiah C, Chen MH, et al. Harassment and discrimination in medical training: a systematic review and meta-analysis. Acad Med. 2014;89(5):817-827. doi:10.1097/ACM.0000000000000200

2. Carr PL, Ash AS, Friedman RH, et al. Faculty perceptions of gender discrimination and sexual harassment in academic medicine. Ann Intern Med. 2000;132(11):889-896. doi:10.7326/0003-4819-132-11200006060-00007

3. Carr PL, Gunn CM, Kaplan SA, Raj A, Freund KM. Inadequate progress for women in academic medicine: findings from the National Faculty Study. J Womens Health (Larchmt). 2015;24 (3):190-199. doi:10.1089/jwh.2014.4848

4. van Ryn M, Fu SS. Paved with good intentions: do public health and human service providers contribute to racial/ethnic disparities in health? Am J Public Health. 2003;93(2):248-255. doi:10.2105/ AJPH.93.2.248

5. Croskerry P. The importance of cognitive errors in diagnosis and strategies to minimize them. Acad Med. 2003;78(8):775-780. doi:10.1097/00001888-200308000-00003

6. Freund KM, Raj A, Kaplan SE, et al. Inequities in academic compensation by gender: a follow-up to the national faculty survey cohort study. Acad Med. 2016;91(8):1068-1073. doi:10.1097/ACM.000 0000000001250
7. Jagsi R, Griffith KA, Stewart A, Sambuco D, DeCastro R, Ubel PA. Gender differences in the salaries of physician researchers. JAMA. 2012;307(22):2410-2417. doi:10.1001/jama.2012.6183

8. Raj A, Carr PL, Kaplan SE, Terrin N, Breeze JL, Freund KM. Longitudinal analysis of gender differences in academic productivity among medical faculty across 24 medical schools in the United States. Acad Med. 2016;91(8):1074-1079. doi:10.1097/ACM.000000000 0001251

9. Foote MB, DeFilippis EM, Rome BN, Divakaran S, Yialamas MA. Use of "doctor" badges for physician role identification during clinical training. JAMA Intern Med. 2019;179:1582. doi:10.1001/ jamainternmed.2019.2416

10. Rotenstein LS, Berman RA, Katz JT, Yialamas MA. Making the voices of female trainees heard. Ann Intern Med. 2018;169 (5):339-340. doi:10.7326/M18-1118

11. Jagsi R, Griffith KA, Jones R, Perumalswami CR, Ubel P, Stewart A. Sexual harassment and discrimination experiences of academic medical faculty. JAMA. 2016;315(19):2120-2121. doi:10.1001/jama.2016.2188

12. Denton GD, Papp KK. Tackling some wicked problems in medical education. J Gen Intern Med. 2019;34(5):652-653. doi:10.1007/ s11606-019-04930-x

13. Yialamas MA. Women's leadership and gender bias curriculum for internal medicine residents. Proceedings from the American Medical Women's Association Graduate Medical Education Symposia: the first three years, 2018-2020. Perm J. 2020;47-48.
Advances in Medical Education and Practice

\section{Publish your work in this journal}

Advances in Medical Education and Practice is an international, peerreviewed, open access journal that aims to present and publish research on Medical Education covering medical, dental, nursing and allied health care professional education. The journal covers undergraduate education, postgraduate training and continuing medical education

\section{Dovepress}

including emerging trends and innovative models linking education, research, and health care services. The manuscript management system is completely online and includes a very quick and fair peer-review system. Visit http://www.dovepress.com/testimonials.php to read real quotes from published authors. 